\title{
First Clinical Data of Pressurized Intraperitoneal Aerosol Chemotherapy (PIPAC) as Salvage Therapy for Peritoneal Metastatic Biliary Tract Cancer
}

\author{
THOMAS A. FALKENSTEIN ${ }^{1 *}$, THORSTEN O. GÖTZE ${ }^{2 *}$, MEHDI OUAISSI ${ }^{3}$, \\ CLEMENS B. TEMPFER ${ }^{4,5}$, URS GIGER-PABST ${ }^{1,5}$ and CÉDRIC DEMTRÖDER ${ }^{5}$ \\ ${ }^{1}$ Basic Research Laboratories of the Department of Surgery, ${ }^{4}$ Department of Obstetrics and Gynecology, and \\ ${ }^{5}$ General Surgery and Therapy Center for Peritoneal Carcinomatosis, \\ St. Mary's Hospital, Ruhr University Bochum, Herne, Germany; \\ ${ }^{2}$ Institute of Clinical Cancer Research, UCT-University Cancer Center Frankfurt, \\ Hospital Nordwest, Frankfurt, Germany; \\ ${ }^{3}$ Department of Digestive, Oncology, Endocrine and Liver Transplantation, \\ Colorectal Surgery Unit, Trousseau Hospital, Tours, France
}

\begin{abstract}
Background: Patients suffering from peritoneal metastasis of biliary tract cancer were treated with pressurized intraperitoneal aerosol chemotherapy (PIPAC). Patients and Methods: This was a study carried out at a single institution, tertiary referral center certified for therapy of peritoneal disease. Retrospective data analysis was performed of prospective data for PIPAC with intraperitoneal low-dose doxorubicin $\left(1.5 \mathrm{mg} / \mathrm{m}^{2}\right)$ and cisplatin $\left(7.5 \mathrm{mg} / \mathrm{m}^{2}\right)$ delivered at intervals of 6 weeks. The outcome criteria were microscopic pathological response, survival, and adverse events [Common Terminology Criteria of Adverse Events (v4.0)]. Results: A total of 13 patients ( male/female $=8 / 5)$ with a mean age of $58($ range $=37-75)$ years underwent 17 PIPAC procedures without intraoperative complications. The mean number of PIPAC applications was 1.3 (range=0-3). Due to non-accessibility of the abdominal cavity in two patients (15.4\%) and rapid clinical deterioration in six patients (46\%), five patients underwent two or more PIPAC applications and were, therefore, eligible for histological analysis to assess
\end{abstract}

\footnotetext{
*These Authors contributed equally to this study.

Correspondence to: Giger-Pabst Urs, MD, Department of General Surgery \& Therapy Center for Peritoneal carcinomatosis, St. Mary's Hospital Herne, University Hospital of Ruhr University Bochum, Hölkeskampring 40, D-44625 Herne, Germany. Tel: +49 023234995893, e-mail: urs.pabst@elisabethgruppe.de
}

Key Words: PIPAC, biliary tract, gallbladder, cancer, peritoneal metastasis, tumor regression. carcinoma regression. Overall tumor regression of any degree was determined in 4/5 patients. An overall median survival of 85 days (95\% confidence interval $(C I)=59.2-110.4$ days) after the first PIPAC application was observed. No complications greater than Common Terminology Criteria of Adverse Events (v4.0) level 2 occurred. Conclusion: PIPAC can induce objective regression of systemic chemotherapyresistant peritoneal metastasis of biliary tract cancer. However, due to a rapid clinical deterioration of the patients, almost two-thirds of the patients cannot undergo repetitive PIPAC courses.

Biliary tract cancer (BTC) is in most cases adenocarcinoma arising from the epithelial lining of the gallbladder and the intra- and extrahepatic (hilar and distal common bile duct) bile ducts. Gallbladder carcinoma (GBC) is the fifth most common neoplasm of the digestive tract and has an overall incidence of 3 per 100,000 people (1). In addition, there are carcinomas of the bile ducts [intra- and extrahepatic cholangiocarcinoma (ICC and ECC)]. In Germany, approximately 2,000 new GBC cases and 3,000 new ECC cases were diagnosed in 2012 (2). Complete surgical resection offers the only chance for cure. Unfortunately, most patients are diagnosed at an advanced tumor stage so that only $20-35 \%$ of patients can undergo curative surgery. However, even after curative surgery, the prognosis remains poor and recurrence rates are high (3). Therefore, most patients will be managed with palliative systemic chemotherapy. For patients with advanced BTCs treated with systemic first-line chemotherapy, the reported median overall survival is around 12 months (4). The peritoneum is a frequent site of metastatic disease and in the case of the 
presence of peritoneal metastasis (PM), a median survival time of 4.8 months is reported $(5,6)$.

Pressurized intraperitoneal aerosol chemotherapy (PIPAC) is a new technique for delivering intra-peritoneal chemotherapy to treat PM. During standard diagnostic laparoscopy, liquid chemotherapy drugs are delivered as a pressurized aerosol into the abdominal cavity. This approach allows administration of the smallest amounts of chemotherapy at high concentrations into the abdominal cavity. The pressure of the capnoperitoneum is intended to additionally enhance the cytotoxic effect of PIPAC (7). The safety, feasibility and antitumoral effect of PIPAC on systemic chemoresistant PM have already been reported by several independent groups of surgical oncologists and gynecologists (8-12). However, no data about PIPAC to treat PM of BTCs has been reported to our knowledge.

\section{Patients and Methods}

Patients and regulatory framework. Since April 2012, PIPAC therapy has been applied with approved drugs for i.v. therapy as offlabel use. Each patient was evaluated at a multidisciplinary Tumor Board. The indication for PIPAC therapy was decided on a case-bycase basis. Patients with progressive PC after or under evidencebased systemic chemotherapy or patients who did not qualify for systemic chemotherapy due to medical contraindications underwent PIPAC. There were no strict exclusion criteria for PIPAC, but patients suffering from clinical signs of gastrointestinal occlusion or with a Karnofsky Index less than 50\%; [Eastern Cooperative Oncology Group Performance Score (ECOG PS) >3] were excluded. We intended to deliver at least three PIPAC cycles separated by a 6-week time interval. In patients undergoing systemic chemotherapy simultaneously with PIPAC, systemic therapy was delivered in the interval between two PIPAC sessions. The study was performed in line with the guidelines of the Declaration of Helsinki, and each patient was asked to give their written informed consent for data collection, as well as for publication of data in an anonymous manner. Data collection and analysis within a tumor registry were performed with the approval of the local Institutional Review Board (Ethics Committee of the Ruhr University Bochum, Germany; \#15-5280)

PIPAC procedure. The standard PIPAC procedure has been described in detail elsewhere $(7,10-12)$. The access to the abdominal cavity was usually obtained via a mini laparotomy lateral to the left rectus muscle in the midclavicular line at the level of the umbilicus. Peritoneal biopsies from all four abdominal quadrants (if possible) were retrieved and sent for histological analysis. Doxorubicin at a dose of $1.5 \mathrm{mg} / \mathrm{m}^{2}$ body surface area (BSA) in 50 $\mathrm{ml}$ of $0.9 \% \mathrm{NaCl}$ followed by cisplatin at a dose of $7.5 \mathrm{mg} / \mathrm{m}^{2}$ BSA in $150 \mathrm{ml}$ of $0.9 \% \mathrm{NaCl}$ were aerosolized. All PIPAC procedures were performed by senior surgeons trained in PIPAC technology.

Data collection follow-up and statistical analysis. Data were collected by clinical study nurses within a prospective data registry. Follow-up data were obtained by telephone calls until death occurred or until last documentation at 20th June 2017. Histological tumor response was assessed by an independent pathological reference center (Institute of Pathology, Ruhr University Bochum, Bochum,
Table I. Baseline characteristics of patients undergoing pressurized intraperitoneal aerosol chemotherapy (PIPAC).

\begin{tabular}{|c|c|c|}
\hline Characteristic & No. & $(\%)$ \\
\hline Total patients & 13 & 100 \\
\hline \multicolumn{3}{|l|}{ Gender } \\
\hline Male & 8 & 62 \\
\hline Female & 5 & 38 \\
\hline \multicolumn{3}{|l|}{ Age (years) } \\
\hline$<50$ & 3 & 23 \\
\hline $50-60$ & 5 & 38.5 \\
\hline$>60$ & 5 & 38.5 \\
\hline \multicolumn{3}{|l|}{ Primary tumor site } \\
\hline GB & 5 & 38.5 \\
\hline $\mathrm{IHC}$ & 3 & 23 \\
\hline EHC & 5 & 38.5 \\
\hline \multicolumn{3}{|l|}{ Prior surgery } \\
\hline Yes (R0 resection) & 6 & 46 \\
\hline Yes (R1 resection) & 3 & 23 \\
\hline No & 4 & 31 \\
\hline No systemic chemotherapy & 6 & 46 \\
\hline Prior first-line chemotherapy & 7 & 54 \\
\hline Gemcitabine \& cisplatin & 5 & 38.5 \\
\hline Gemcitabine \& oxaliplatin & 1 & 7.7 \\
\hline Gemcitabine mono therapy & 1 & 7.7 \\
\hline \multicolumn{3}{|l|}{ Best response to first-line chemotherapy } \\
\hline Partial response & 2 & 15.4 \\
\hline Stable disease & 2 & 15.4 \\
\hline Progressive disease & 3 & 23 \\
\hline Second-line chemotherapy & 4 & 31 \\
\hline FOLFOX & 2 & 15.4 \\
\hline XELIRI & 1 & 7.7 \\
\hline 5FU monotherapy & 1 & 7.7 \\
\hline Second-line chemotherapy \& PIPAC & 3 & 23 \\
\hline PIPAC without any systemic chemotherapy & 10 & 77 \\
\hline \multicolumn{3}{|l|}{ Performance status (ECOG) } \\
\hline 0 or 1 & 6 & 46 \\
\hline 2 or 3 & 7 & 54 \\
\hline \multicolumn{3}{|l|}{ Metastatic sites } \\
\hline Peritoneal & 13 & 100 \\
\hline Extra-peritoneal (pleura) & 2 & 15.4 \\
\hline
\end{tabular}

GB: Gallbladder; IHC: intra-hepatic cholangiocarcinoma; EHC: extrahepatic cholangiocarcinoma.

Germany). To evaluate the histological tumor regression grade (TRG) induced by PIPAC, the following criteria according to Dworak et al. were applied: TRG 0: No regression; TRG 1: predominantly tumor with significant fibrosis with/without vasculopathy; TRG 2: predominantly tumor with significant fibrosis with scattered tumor cells (slightly recognizable histologically); TRG 3: only scattered tumor cells in the space of fibrosis with/without acellular mucin; TGR 4: no vital tumor cells detectable (13). In this study, whenever different scores were found in different tissue samples of the same patient, the lowest TRG value was reported. Adverse events were graded according to the Common Terminology Criteria for Adverse Events (v4.0) (14). Data analysis was conducted retrospectively and data are given as absolute numbers, percentage, mean (range: minimum to maximum) or median with interquartile 


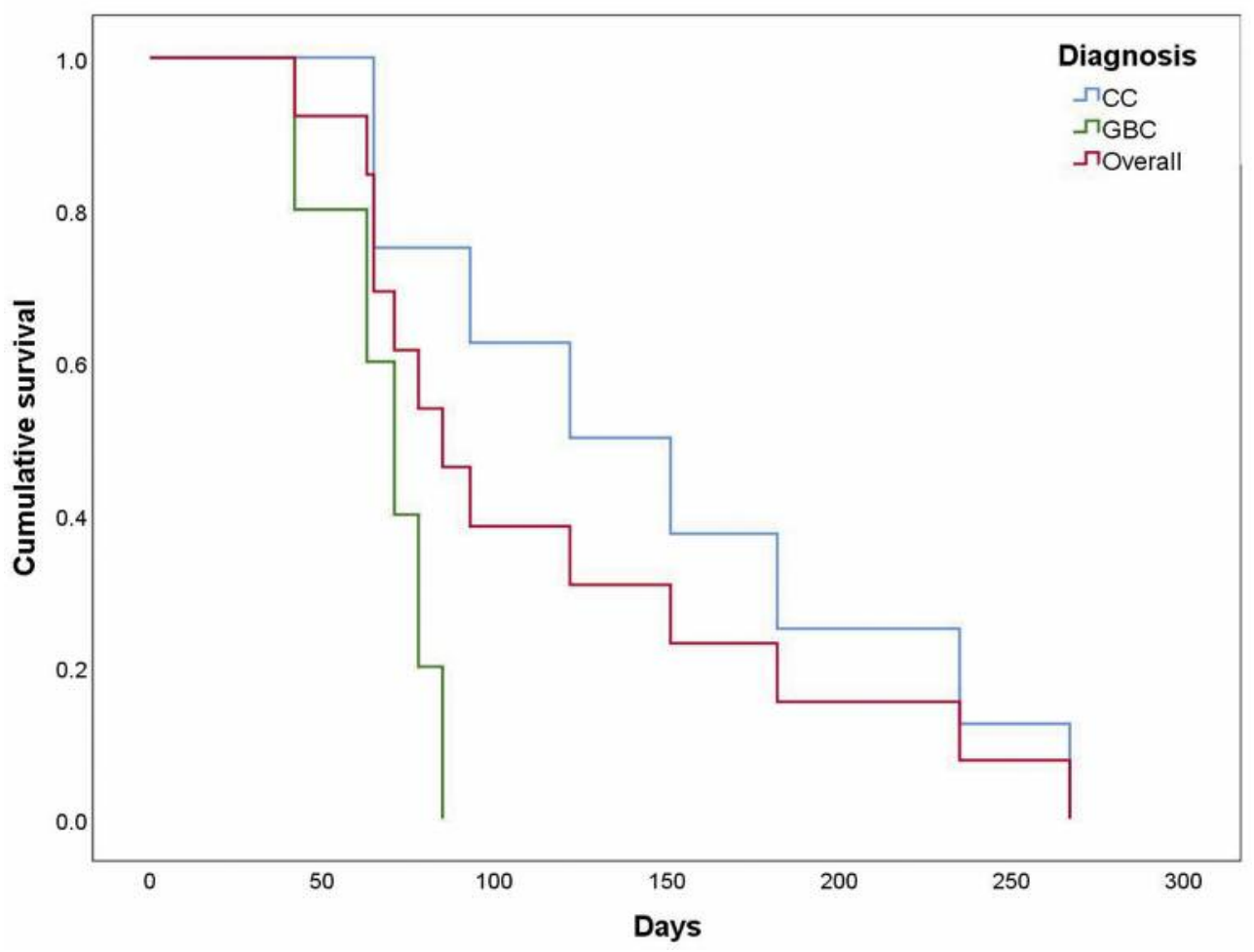

Figure 1. Kaplan-Meier curve of overall survival after first pressurized intraperitoneal aerosol chemotherapy application according to diagnosis. CC: Intra- and extrahepatic cholangiocarcinoma; GBC: gallbladder cancer.

range (IQR). Overall median [95\% confidence interval (CI)] survival was modelled using a Kaplan-Meier curve with SPSS ${ }^{\circledR}$ Statistics 25 (IBM Corp., Armonk, NY, USA).

\section{Results}

Between February 2013 and January 2017, a total of 13 patients (male/female $=8 / 5$ ) with a mean age of 58 (range $=37-75$ ) years and a median Karnofsky Index of $70 \%$ (IQR=60-85\%) were scheduled for PIPAC. The primary tumor location was the gallbladder in five, intra-hepatic in three and extra-hepatic cholangiocarcinoma in five. Additionally, two patients suffered from histologically documented metastatic spread to the thoracic cavity. Nine patients had initially undergone extensive curative surgery whereas five patients were diagnosed with synchronous PM during staging laparoscopy or laparotomy. Seven patients had progressive peritoneal carcinomatosis under or after a minimum of one line of systemic chemotherapy, six patients, due to medical contraindications, were systemic chemotherapy-naive. Three patients received systemic chemotherapy and PIPAC applications, whereas 10 patients had PIPAC treatment only. The average time interval between the diagnosis of PM and the first PIPAC application was 190 (range $=15-930$ ) days. Patient demographic data as well as details on previous surgical procedures and systemic chemotherapy are summarized in Table I.

Access to the abdominal cavity for the first PIPAC application failed in two patients $(2 / 13)$, representing a primary non-accessibility rate of $15.4 \%$. In the 11 patients who received a first PIPAC cycle, a median Sugarbaker's peritoneal carcinomatosis index (PCI) score of 20 (IQR=827 ) and malignant ascites of $2800(\mathrm{IQR}=1200-6000) \mathrm{ml}$ were documented.

The mean number of PIPAC applications was 1.3 (range $=0-3$, whereas one patient received three applications, four patients two, six patients one and two patients none. The reasons for the premature ending of PIPAC treatment (three cycles intended) were ileus in three patients $(23.1 \%)$, clinical deterioration/cachexia syndrome in four $(30.8 \%)$, septic complications/fulminant pneumonia in three $(23.1 \%)$ and cardiac decompensation in one patient $(7.7 \%)$. On an intention-to-treat analysis, one patient $(1 / 13=7.7 \%)$ had three PIPAC applications.

A total of 17 successful PIPAC procedures were delivered. The mean procedure time was 89.0 (range=72-107) minutes with no observed intraoperative complications. Seven patients $(7 / 17,41.4 \%)$ suffered from mild postoperative complications (Common Terminology Criteria for Adverse Events grade 1). 
Table II. Individual patient demographic data and details on previous surgical interventions and systemic chemotherapy treatment prior to pressurized intraperitoneal aerosol chemotherapy (PIPAC).

\begin{tabular}{|c|c|c|c|c|c|c|c|c|c|c|c|c|c|}
\hline \multirow[b]{3}{*}{ Patient no. } & \multicolumn{12}{|c|}{ PIPAC } & \multirow[b]{3}{*}{ Comment } \\
\hline & \multicolumn{4}{|c|}{1} & \multicolumn{4}{|c|}{2} & \multicolumn{4}{|c|}{3} & \\
\hline & PCI & $\begin{array}{l}\text { Ascites } \\
(\mathrm{ml})\end{array}$ & CTCAE & TRG & PCI & $\begin{array}{c}\text { Ascites } \\
(\mathrm{ml})\end{array}$ & CTCAE & TRG & PCI & $\begin{array}{l}\text { Ascites } \\
(\mathrm{ml})\end{array}$ & CTCAE & TRG & \\
\hline 1 & 31 & 2000 & 0 & 0 & & & & & & & & & $\begin{array}{l}\text { Clinical deterioration; died } \\
65 \text { days after 1st PIPAC }\end{array}$ \\
\hline 2 & 20 & 2000 & & 0 & & & & & & & & & $\begin{array}{l}\text { Pneumonia and sepsis; died } \\
71 \text { days after 1st PIPAC }\end{array}$ \\
\hline 3 & 18 & 6000 & 2 & 0 & 22 & 7000 & 2 & 0 & & & & & Ileus; died 85 days after 1st PIPAC \\
\hline 4 & 8 & 7000 & 2 & 0 & & & & & & & & & $\begin{array}{c}\text { Tumor cachexia; died } 63 \\
\text { days after 1st PIPAC }\end{array}$ \\
\hline 5 & 5 & 1200 & 1 & 0 & 1 & 500 & 1 & 1 & & & & & $\begin{array}{l}\text { Clinical deterioration; died } \\
78 \text { days after 1st PIPAC }\end{array}$ \\
\hline 6 & & Prim & ary non-a & iccessib & fility o & $f$ the $a b d$ & ominal cav & vity & & & & & Ileus; died 151 days after 1st PIPAC \\
\hline 7 & 27 & 5000 & 1 & 0 & & & & & & & & & $\begin{array}{l}\text { Duodenal stenosis, stent placement; } \\
\text { died } 42 \text { days after } 1 \text { st PIPAC }\end{array}$ \\
\hline 8 & & Prim & ary non-a & iccessib & fility o & $f$ the $a b d$ & ominal cav & vity & & & & & $\begin{array}{c}\text { Biliary sepsis; died } 267 \text { days } \\
\text { after 1st PIPAC }\end{array}$ \\
\hline 9 & 15 & 100 & 1 & 0 & 13 & 100 & 1 & 2 & & & & & $\begin{array}{c}\text { Clinical deterioration; died } 235 \\
\text { days after 1st PIPAC }\end{array}$ \\
\hline 10 & 20 & 200 & 1 & 0 & 18 & 2500 & 1 & 3 & & & & & $\begin{array}{c}\text { Pneumonia; died } 182 \text { days } \\
\text { after 1st PIPAC }\end{array}$ \\
\hline 11 & 26 & 2800 & 2 & 0 & & & & & & & & & $\begin{array}{c}\text { Biliary sepsis, died } 93 \text { days } \\
\text { after 1st PIPAC }\end{array}$ \\
\hline 12 & 32 & 5000 & 2 & 0 & & & & & & & & & $\begin{array}{c}\text { Portal hypertension and } \\
\text { cardiac decompensation; } \\
\text { died } 65 \text { days after 1st PIPAC }\end{array}$ \\
\hline 13 & 7 & 8000 & 2 & 0 & 4 & 5000 & 1 & 1 & 3 & 3000 & 0 & 2 & Ileus; died 122 days after 1st PIPAC \\
\hline
\end{tabular}

TRG: Tumor regression grade: 0: no regression 1: predominantly tumor with significant fibrosis with/without vasculopathy 2: predominantly fibrosis with scattered tumor cells (slightly recognizable histologically), 3: only scattered tumor cells in the space of fibrosis with/without acellular mucin 4: no vital tumor cells detectable; PCI: Sugarbaker peritoneal carcinomatosis index; N/A: not applicable; CTCEA: Common Terminology Criteria of Adverse Events (v4.0)

Transient prerenal kidney toxicity (Common Terminology Criteria for Adverse Events grade 2; creatinine 2.0-3.0 $\times$ above baseline) was observed after 6/17 (35.3\%) interventions. No Common Terminology Criteria for Adverse Events grade 3 or 4 complications occurred. There was no PIPAC-related mortality.

Out of 13 patients, five received two or more PIPAC cycles and were therefore eligible for histological TRG analysis. The median number of biopsy samples harvested during each PIPAC session was six (IQR=4-8). A local peritonectomy of $3 \times 3 \mathrm{~cm}$ was obtained in every case. No tumor regression (TRG 0 ) was observed in one patient (1/5). A total of two patients (2/5) showed minimal or moderate histological tumor regression (TRG 1 and 2). In another two patients (2/5), extensive tumor regression (TRG 3) was observed. Overall, repetitive PIPAC applications induced histological tumor regression in 4/5 (80\%) patients.
An overall median survival time of 85 days $(95 \%$ $\mathrm{CI}=59.2-110.4$ days) after the first PIPAC application was noted. According to the primary tumor site, the median overall survival for patients with GBC was 71.0 (95\% $\mathrm{CI}=53.8-88.2)$ days and for those with ICC/ECC 122.0 (95\% $\mathrm{CI}=41.6-202.4)$ days. All patients died during the follow-up period from February 2013 to June 2017. Details about PIPAC therapy, perioperative complications, and histological regression are summarized in Table II. Survival data after the first PIPAC cycle are given in Figure 1.

\section{Discussion}

The dismal prognosis and the high frequency of PM as site of disease manifestation of BTC emphasizes the need to improve patient outcomes by intensifying the treatment of PM. 
PIPAC is a new technique for treating end-stage PM by delivering intraperitoneal chemotherapy as a pressurized aerosol. Low-dose liquid chemotherapy drugs are nebulized into the abdominal cavity during a standard laparoscopic procedure by means of a spraying device connected to a high-pressure injector (7). Based on retrospective cohort data and a phase II study, there is growing evidence that this approach is feasible and safe with only minimal local or systemic side-effects. Objective histological tumor regression in systemic chemoresistant peritoneal carcinomatosis of ovarian, gastric, colorectal and, very recently, pancreatic cancer in $50 \%$ to $70 \%$ of patients was observed (8-12).

In the present study, no intraoperative complications occurred. The postoperative side-effects were minimal Nevertheless, due to large amounts of ascites evacuated before delivering PIPAC, transient prerenal renal failure, which required careful perioperative volume resuscitation, was observed after more than one-third of all PIPAC applications. However, no severe adverse events (more than grade 2 by Common Terminology Criteria for Adverse Events) were observed.

All treatment parameters of PIPAC, such as the minimum number of PIPAC applications to induce maximum tumor regression, are still empirical with limited available evidence. It is assumed by most pioneers of PIPAC therapy that least three PIPAC cycles are probably required to induce a substantial regression of PM. Previous data of PM of gastric, colorectal and ovarian origin treated with repetitive PIPAC applications documented the feasibility of such an approach with an average number of 2.5 to 2.8 PIPAC applications per patient (9-12). However, these findings are in contrast to our present data where only 1.3 PIPAC applications were successfully delivered per patient. Since the primary nonaccess rate of $15.4 \%$ is within the range of previous reports (12), the main reason for premature ending of PIPAC therapy in our small case series was related to the rapid clinical deterioration of the patients' general condition. This fact is also reflected by the poor median overall survival of only 3 months after the first PIPAC treatment. We are aware that our data are difficult to interpret but they are probably best discussed in the context of those of second-line systemic chemotherapy. Currently, data about systemic second-line chemotherapy for BTCs report a median overall survival of around 7 months (15). This is more than twice as long as our current findings for PIPAC. At first glance, PIPAC treatment appears to be poorer in terms of survival. However, in regard to our present case series, it must be taken into account that there are significant differences regarding the patient selection between PIPAC and second-line chemotherapy. In a recent study by Brieau et al., only $40 \%$ of patients who underwent second-line chemotherapy for BTCs suffered from documented PM, whereas a high number of cases $(70 \%)$ had an excellent physical performance status (ECOG PS 0-1) (16).
In contrast to this, almost $80 \%$ of the patients managed with PIPAC in our study were unable to receive any systemic treatment. Furthermore, almost two-thirds of the patients were in a poor physical condition, with an ECOG PS of 2-3.

Today, the efficacy of second-line chemotherapy is still under debate, but current data indicate that patients in a good physical condition (ECOG PS 0-1) and a documented response to an evidence-based first-line chemotherapy are likely to benefit from second-line chemotherapy $(16,17)$.

Based on such findings it must be stated that the observed poor survival in our present case series of patients managed with PIPAC is very likely due to patient selection. In view of the available data, PIPAC might best be delivered in combination with systemic second-line chemotherapy in patients eligible for second-line chemotherapy rather than as a single salvage therapy for those patients who are not candidates for systemic chemotherapy. Such an approach is supported by our observation that an objective histological regression of PM was documented in $80 \%$ of cases after repeated PIPACs. However, whether such an approach can improve the clinical outcome of such patients remains unclear.

\section{Conclusion}

In this first small case series of patients suffering from PM of BTC, repetitive PIPAC application, due to rapid clinical deterioration, failed in the majority of our patients. Nevertheless, in those patients who received at least two PIPAC applications, histological regression of PM was documented in $80 \%$ of them. Based on our early data, we conclude that PIPAC therapy should be initiated at an earlier stage of the disease. Thus, the number of patients eligible for multiple PIPAC administrations may be increased. Improved patient selection may also be based on known positive prognostic factors for a response to second-line chemotherapy. A combination of systemic second-line chemotherapy with repetitive PIPAC applications appears to be promising.

\section{Conflict of Interests}

This study was funded by institutional funds. Falkenstein TA, Götze TO, Ouaissi M, Tempfer CB, Giger-Pabst U and Demtröder C have no conflicts of interest or financial ties to disclose.

\section{Acknowledgements}

The Authors are thankful to Mrs. J. Kosel (research assistant), Mrs. J. Koke (study nurse) and Karla Nemet (student) for data collection.

\section{References}

1 Goetze TO: Gallbladder carcinoma: Prognostic factors and therapeutic options. World J Gastroenterol 21(43): 12211-12217, 2015. 
2 Gall bladder and biliary tract. In: Cancer in Germany 2011/2012 10th Edition. Robert Koch Institute (ed.) and the Association of Population-based Cancer Registries in Germany (ed.). Berlin, pp. 46-49, 2016.

3 Zhu AX, Hong TS, Hezel AF and Kooby DA: Current management of gallbladder carcinoma. Oncologist 15(2): 168181,2010

4 Valle J, Wasan H, Palmer DH, Cunningham D, Anthoney A, Maraveyas A, Madhusudan S, Iveson T, Hughes S, Pereira SP, Roughton $\mathrm{M}$ and Bridgewater J: ABC-02 Trial Investigators. Cisplatin plus gemcitabine versus gemcitabine for biliary tract cancer. N Engl J Med 362(14): 1273-1281, 2010.

5 Nishio H, Nagino M, Ebata T, Yokoyama Y, Igami T and Nimura Y: Aggressive surgery for stage IV gallbladder carcinoma; what are the contraindications? J Hepatobiliary Pancreat Surg 14(4): 351-357, 2007.

6 Maplanka C: Gallbladder cancer, treatment failure and relapses: the peritoneum in gallbladder cancer. J Gastrointest Cancer 45(3): 245-255, 2014.

7 Solass W, Kerb R, Mürdter T, Giger-Pabst U, Strumberg D, Tempfer C, Zieren J, Schwab $M$ and Reymond MA: Intraperitoneal chemotherapy of peritoneal carcinomatosis using pressurized aerosol as an alternative to liquid solution: first evidence for efficacy. Ann Surg Oncol 21(2): 553-559, 2014.

8 Grass F, Vuagniaux A, Teixeira-Farinha H, Lehmann K, Demartines N and Hübner M: Systematic review of pressurized intraperitoneal aerosol chemotherapy for the treatment of advanced peritoneal carcinomatosis. Br J Surg 104(6): 669-678, 2017.

9 Graversen M, Detlefsen S, Bjerregaard JK, Pfeiffer P and Mortensen MB: Peritoneal metastasis from pancreatic cancer treated with pressurized intraperitoneal aerosol chemotherapy (PIPAC). Clin Exp Metastasis 2017. doi: 10.1007/s10585-0179849-7. [Epub ahead of print]

10 Demtröder C, Solass W, Zieren J, Strumberg D, Giger-Pabst U and Reymond MA: Pressurized intraperitoneal aerosol chemotherapy with oxaliplatin in colorectal peritoneal metastasis. Colorectal Dis 18(4): 364-371, 2016.
11 Nadiradze G, Giger-Pabst U, Zieren J, Strumberg D, Solass W and Reymond MA: Pressurized intraperitoneal aerosol chemotherapy (PIPAC) with low-dose cisplatin and doxorubicin in gastric peritoneal metastasis. J Gastrointest Surg 20(2): 367373, 2016.

12 Tempfer CB, Rezniczek GA, Ende P, Solass W and Reymond MA: Pressurized intraperitoneal aerosol chemotherapy with cisplatin and doxorubicin in women with peritoneal carcinomatosis: A Cohort Study. Anticancer Res 35(12): 67236729, 2015.

13 Dworak O, Keilholz L and Hoffmann A: Pathological features of rectal cancer after preoperative radiochemotherapy. Int $\mathbf{J}$ Colorectal Dis 12(1): 19-23, 1997.

14 Common Terminology Criteria for Adverse Events (CTCAE), Version 4.0. U.S: Department of Health and Human Services. National Institutes of Health, National Cancer Institute, Published: May 28, 2009 (v4.03: June 14, 2010).

15 Lamarca A, Hubner RA, David Ryder W and Valle JW: Secondline chemotherapy in advanced biliary cancer: a systematic review. Ann Oncol 25(12): 2328-2338, 2014.

16 Brieau B, Dahan L, De Rycke Y, Boussaha T, Vasseur P, Tougeron D, Lecomte T, Coriat R, Bachet JB, Claudez P, Zaanan A, Soibinet P, Desrame J, Thirot-Bidault A and Trouilloud I, Mary F, Marthey L, Taieb J, Cacheux W and Lièvre A: Secondline chemotherapy for advanced biliary tract cancer after failure of the gemcitabine-platinum combination: A large multicenter study by the Association des Gastro-Entérologues Oncologues. Cancer 121(18): 3290-3297, 2015.

17 Lee SC, Kim K, Kim H, Kim HJ, Kim SH, Bae SB, Kim CK, Lee N, Lee KT, Park SK, Won JH, Yun J, Hong DS and Park HS: Prognostic factor analysis of second-line chemotherapy in advanced biliary tract cancer. J Clin Oncol 30: abstr e14688, 2012.

Received October 26, 2017

Revised November 14, 2017 Accepted November 16, 2017 